\title{
A Piezo-resistive Application in Textile Design
}

\author{
Mehmet Z. BÍLİR, Banu H. GÜRCÜM \\ Gazi University, Faculty of Art and Design, 06830, Gölbaşl, Ankara, Turkey
}

\begin{abstract}
In this study, it is aimed at that once the user carry loads over his capacity, system gives sound signal as a result work accidents can be decreased as much as possible by using piezo design application. Firstly, circuit design detecting sensor have been made in Proteus-Isis programme. Since parts of circuit can work, transfer the data and give warning signal, software of system have been written in CCS c programme. Piezo system has been explained generally and work principle of design and system data have been shared. It is showed that this prototype study can be applied and it is explained what kind of improvements can be made for future applications.
\end{abstract}

Keywords: Piezo, force sensor, smart textile, piezo sensor.

\section{INTRODUCTION}

Intelligent textiles or products that can react to external conditions such as environment and change their properties or deliver novel uses [1]. Wearable technology and integrated systems have demonstrated during the last 1015 years significant advances in terms of miniaturisation, seamless integration, functionality, comfort, data processing and communication [2]. The first smart textile application was made in 1850 with the aim of electro-therapy as various corsets [3]. The first wearable clothing was made by Edward Thorpe and Claude Shannon in 1955. Curies discovered that some materials could be deformed when they exposed to electric so piezo-textile materials have been started to use [4]. They found that if certain crystals were subjected to mechanical strain, they became electrically polarized and the degree of polarization was proportional to the applied strain. The Curies also discovered that these same materials deformed when they were exposed to an electric field [4].
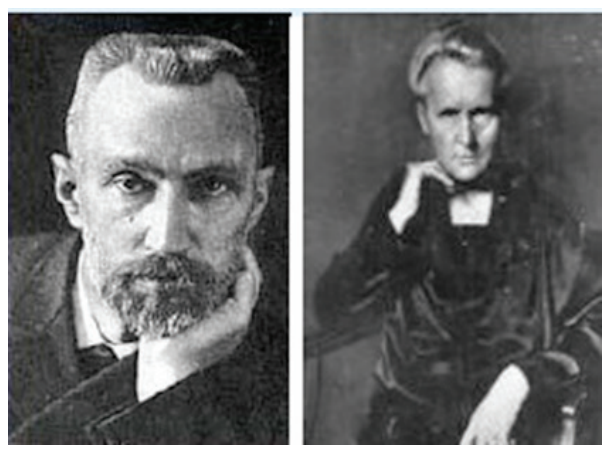

Figure 1. Pierre and Jacques Curie
In 1881, Gabriel Lippman showed theoretically that the reverse mechanism, the inducement of mechanical deformation by polarization is possible. Curie brothers experimentally confirmed deformation of piezoelectric crystals under influence of external electric field [5]. In 1911, piezoelectrics have been used practically first time. In this application, Paul Langevin, a French physicist worked closely with Robert William Boyle, a Canadian physicist, to develop an ultrasonic submarine detector which uses quartz to emit a high frequency chirp. After World War 1, piezoelectric systems were often used in record players, viscosity measuring and finding flaws in materials. In World War II, Fredrick R. Lack of Bell Telephone Laboratories invented the "AT-cut" crystal. "AT-cut" crystals were able to operate using the piezoelectric effect through a much wider range of temperatures. These crystals were used in aviation radio. When pressure (stress) is applied to a material it creates a strain or deformation in the material. In a piezoelectric material strain creates an electrical potential difference (Fig. 2). If we induce some elastic deformation as shown in Figure 3, the symmetry is broken and the three dipole moments no longer cancel - we have polarization induced by mechanical deformation [5]. The effect is reversible, when an electric potential is applied across two sides of a piezoelectric material, it strains. A piezoelectric material cannot be isotropic, or identical in all directions. If there was symmetry in the material there would be no electric polarization yield.

Corresponding Author: Mehmet Z. BİLİR, Tel: +90 (0312) 212 6840, E-mail: mehmetbilir@msn.com

Submitted: 15 October 2014, Revised: 04 March 2015, Accepted: 01 July 2015 


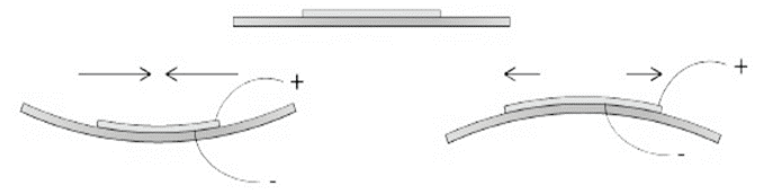

Figure 2. Piezoresistance under pressure [6]

\section{PIEZO FORCE SENSOR}

In this study, we have used FlexiForce A201 Force sensor (Figure 3). This sensor acts as a force sensing resistor in an electrical circuit. When the force sensor is unloaded, its resistance is very high. When a force is applied to the sensor, this resistance decreases. The resistance can be read by connecting a multimeter to the outer two pins, then applying a force to the sensing area.

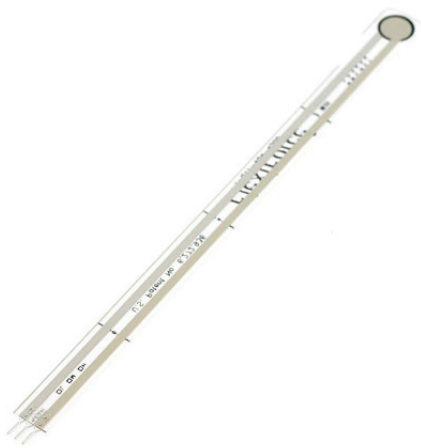

Figure 3. Force Sensor

This sensor have $0.203 \mathrm{~mm}$ thickness, $191 \mathrm{~mm}$ length and $14 \mathrm{~mm}$ width. It can measure 0-100 libres loadings. Sensor contains flexible substrate, silver, pressure sensitive ink, and adhesive layers (Figure 4).

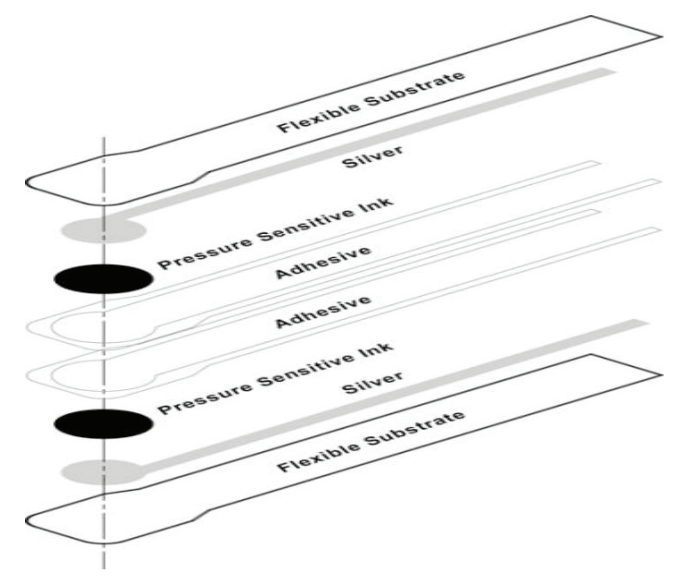

Figure 4. Layers of Force Sensor

\section{CIRCUIT MECHANISM AND DESIGN}

Circuit design have been made by using Proteus Isis programme. Circuit occurs from 14 parts given below in Figure 5.
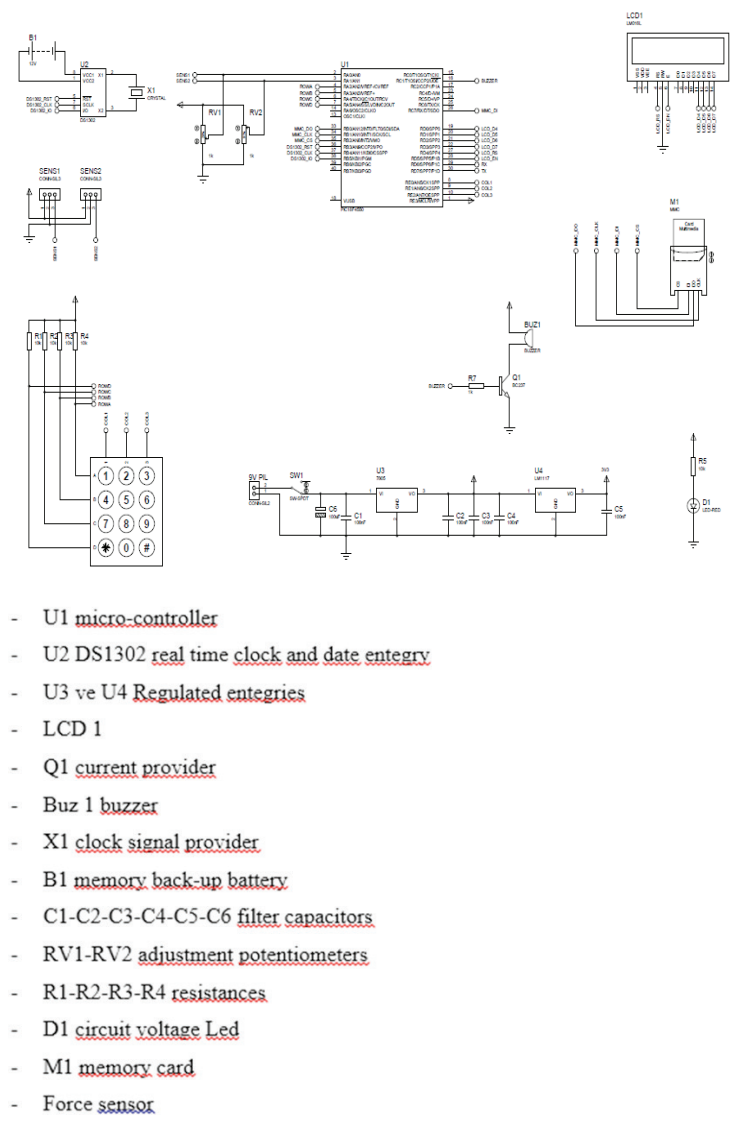

Figure 5. Circuit and parts

This prototype design connects the sensor with a wire. This wire is flexible enough and carries data from sensors to circuit. Circuit mechanism placed on belt or in a bag with user so it can be used easily at the moment of carrying.

First step: First of all, this sensor is so delicate so we try to place it between hard things to feel the load better. For this aim, we have used shoes soles that sensors are glued on carefully (Figure 6).

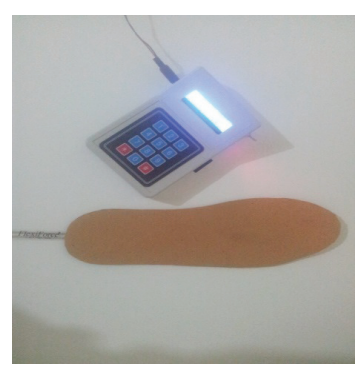

Figure 6. Shoes soles with force sensor 
Second step: Shoes soles with sensors have been places into shoes carefully. In that point, we have lived some problem such as sometimes feet couldn't press the sensor enough because of soft shoes surface, but mostly it worked regularly (Figure 7).

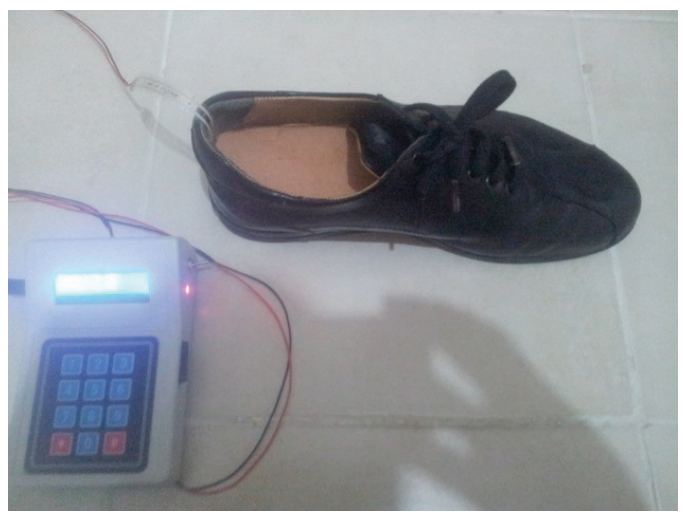

Figure 7. Shoes with sensor

Third step: In this step, our system was ready to use. We wore the shoes and carried load. While carrying load, our machine was saving data so we would be able to see which time how much load pressed on feet (Figure 8).

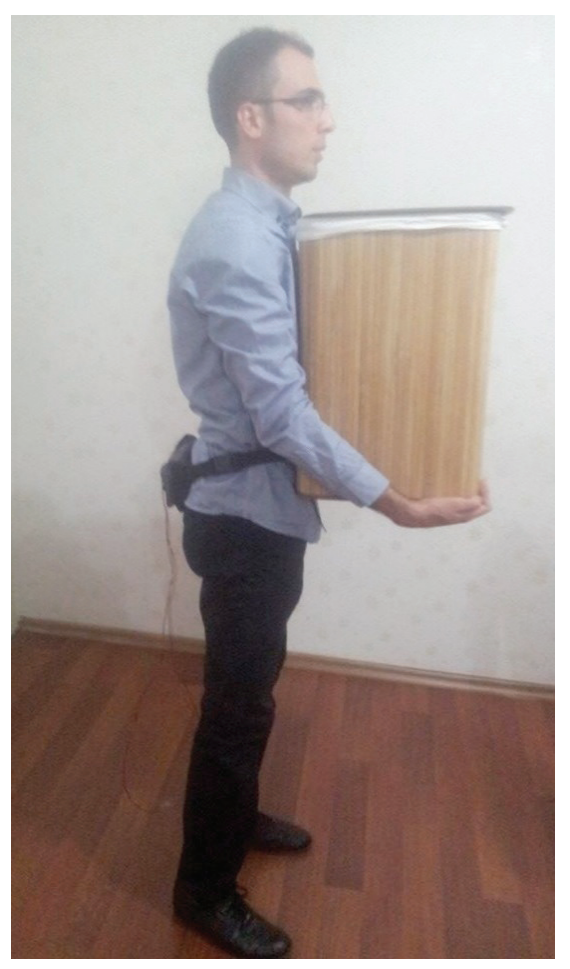

Figure 8. Loading

\section{CONCLUSIONS AND FUTURE WORKS}

System can save data into an SD card so we can use data from pc afterwards. After our working on design, we investigate the some dates (Figure 9). System creates a file named as LOGGER.

From logger data we can see load date, load time and load forces as $\mathrm{kg}$. These data are important for us because if users try to carry any overloading, system saves these data and we can check such conditions and we can warn the worker or user to be more careful for his health. System give sound more signal, if user try to carry heavier loads.

This design is a prototype to make shoes with sensor and it is need to be improved. We plan to improve design with some changes in future with the information that we got from this design. For example;

- Smaller system

- A system that integrated with shoes without any wire

- GPS usage to see data as online

- More advanced sensor

- Flex sensor usage to carry true loading position

- More sensor usage to get more data from balancing problems on foot

\section{REFERENCES}

[1] Karayianni E., (2002). Space technology moves textiles'smart', Smart Materials Bulletin

[2] Bonato P., (2003). Wearable sensors/systems and their impact on biomedical engineering, IEEE Engineering in Medicine and Biology Magazine, pp:18-20.

[3] Fishlock, D., (2001). Doctor volts. IEE Review (5): pp:23-28.

[4] Novetny M. \& Ronkanen P., (2009). Piezoelectric Actuators

[5] Barczy ski R.J., (2011). Piezoelectrics, Politechnika Gda ska, pp:1-20

[6] Gavrilovic B. \& Dhillon J., Piezoelectric Biosensors, Presentation 